\title{
Trend Towards the Use of Complex Networks in Cloud Computing Environment
}

\author{
Mini Singh Ahuja ${ }^{1}$, Randeep Kaur ${ }^{2}$ and Dinesh Kumar ${ }^{3}$ \\ Assistant Professor ${ }^{1}$, student of m.tech ${ }^{2}$, student of m.tech ${ }^{3}$ \\ Department of Computer Science $e^{1,2,3}$, \\ Guru Nanak Dev University Regional campus ${ }^{1,2,}$ SRM university ${ }^{3}$ \\ miniahuja06@gmail.com ${ }^{1}$ er.randeep347@gmail.com ${ }^{2}$,er.dinesh232@ gmail.com ${ }^{3}$
}

\begin{abstract}
As a result of stochasticity of the spreading process and topological complexity of the cloud computing, it is an arduous task to characterize the nodes which are far more influential than others. The entire objective of this paper is to examine complex networking based cloud computing. Complex networks are those networks whose structure is irregular, complex and evolving dynamically. Its main focus may be the analysis of small networks to that particular of system with thousands or millions of node. It has been found from the review that none of the technique is way better for cloud computing. Therefore, this paper ends with the future scope to solve these issues.
\end{abstract}

Keywords: Complex Networks, Cloud Computing.

\section{Introduction}

Complex networks are those networks whose structure is irregular, complex and evolving dynamically [1]. Its main focus could be the analysis of small networks to that of system with thousands or countless node. Complex networks pay more focus on the properties of network of dynamical units. Complex networks can be expanded as networks of networks [3]. The study of complex network is a young and active area of scientific research inspired largely by the empirical study of real-world networks such as computer networks and networks. In complex networks, resource allocation identifies mapping a large quantity of workloads to substrate networks.

Existing heuristic mapping algorithms evaluate the resources of the nodes according to one resource factor or perhaps a product of resource factors that may probably result in an imbalance of the resource allocation. In the cloud computing environment, substrate network identifies the underlying resources, which consists of servers which are interconnected via communication links [1]. Each server has a processing capacity, and each communication link has a bandwidth capacity. Workloads make reference to the service demands of the users made on the cloud. In the cloud computing environment, the workloads are supplied by the cloud users, which are modelled as a group of virtual nodes interconnected via dedicated virtual links.

Cloud Computing is composed by aggregating two terms in the field of technology. First term is Cloud and the second term is computing. Cloud is a swimming of heterogeneous resources. It is a mesh of huge infrastructure and doesn't have relevance using its name "Cloud ;.Infrastructure describes the applications delivered to end users as services within the Internet and the hardware and system software in data centres that's accountable for providing those services. In order to make efficient usage of these resources and ensure their availability to the end users "Computing" is completed centred on certain criteria specified in Service Level Agreement SLA [2]. Infrastructure in the Cloud is made offered to the user's On-Demand basis in pay-as-you-say-manner. 
Computation in cloud is completed with the aim to reach maximum resource utilization with higher availability at minimized cost.

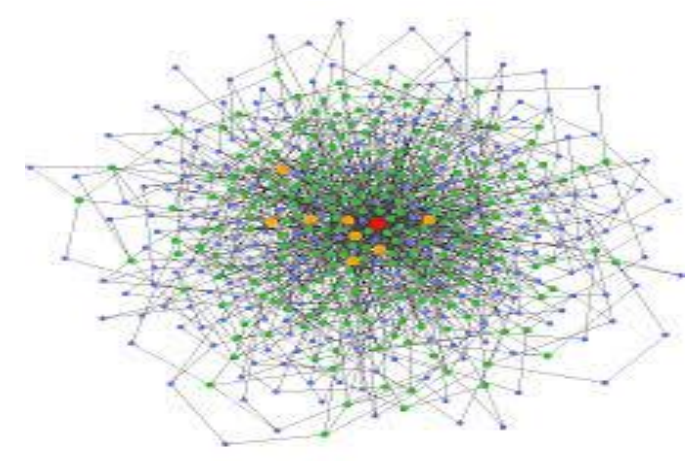

Figure 1. Complex Networks

\section{Basic Concepts of Complex Networks and Cloud Computing}

\subsection{Small-world Networks}

A network with small world effect is any large network with low average path length. Although regular networks and random graphs are generally useful idealizations, many real networks lie somewhere involving the extremes of order and randomness [3]. Watts and Strogatz studied a simple model that can be tuned through the middle ground: a regular lattice. In Watts-Strogatz networks the initial links are replaced by random ones. They unearthed that the slightest bit of rewiring transforms the network in to a 'small world ', with short paths between any two nodes. Yet the network is much more highly clustered than a random graph, in the sense that if $\mathrm{A}$ is connected to $\mathrm{B}$ and $\mathrm{B}$ is connected to $\mathrm{C}$, there is a significantly increased probability that $\mathrm{A}$ will also be connected to $\mathrm{C}$ (a property that's called 'transitivity'). Watts and Strogatz conjectured that the exact same two properties - short paths and high clustering would hold also for most natural and technological networks. Furthermore, they conjectured that dynamical systems coupled this way would display enhanced signal propagation speed and computational power, as weighed against regular lattices of the exact same size [4]. The intuition is that the short paths could provide high-speed communication channels between distant parts of the device, thereby facilitating information flow.

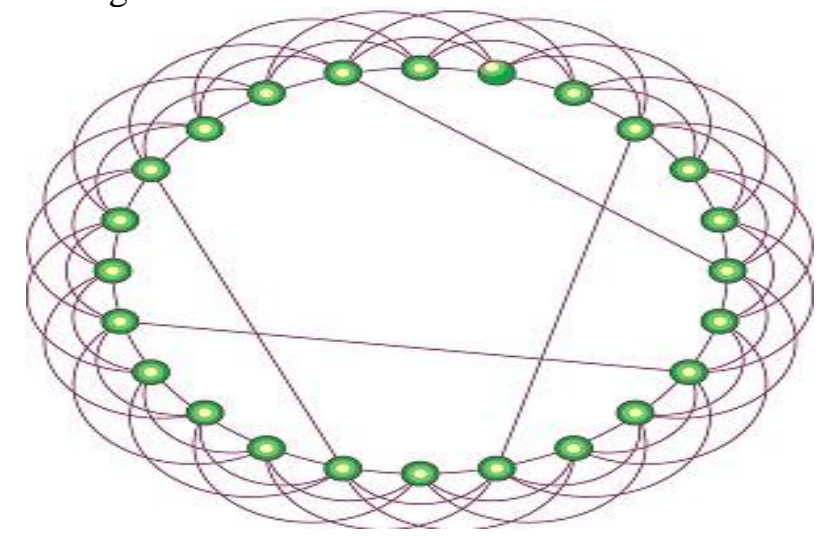

Figure 2. Model of a Small-world Network 


\subsection{Scale-free Networks}

A network is named scale-free if its degree distribution, i.e., the probability that the node selected uniformly randomly includes a certain amount of links (degree), follows a certain mathematical function called the power law. In virtually any real network, some nodes tend to be more highly connected than others. To quantify this effect, let $\mathrm{p}_{\mathrm{k}}$ denote the fraction of nodes that have $\mathrm{k}$ links. Here $\mathrm{k}$ is called the amount and $\mathrm{p}_{\mathrm{k}}$ is the amount distribution [5].

Albert had showed that the heavy-tailed degree distribution emerges automatically from the stochastic growth model in which new nodes are added continuously and attach themselves preferentially to existing nodes, with probability proportional to the amount of the prospective node [6]. Richly connected nodes get richer. More sophisticated models include the consequences of adding or rewiring links, allowing nodes to age so that they may no further accept new links, or varying the shape of preferential attachment. Albert, Jeong and Barabasi suggested that scale-free networks are resistant to random failures because a few hubs dominate their topology. Any node that fails probably has small degree (like most nodes) and so is expendable. The flip side is that such networks are vulnerable to deliberate attacks on the hubs [7].

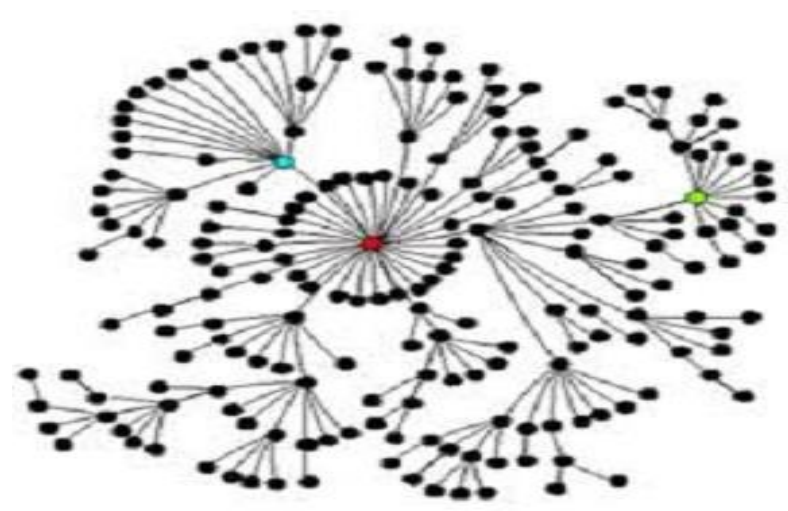

Figure 3. Scale-free Graph, Grown by Attaching New Nodes at Random to Previously Existing Nodes

\section{Load Balancing}

Load balancing in cloud computing provides an efficient treatment for various issues residing in cloud computing environment set-up and usage. Load balancing must take into account two major tasks, one could be the resource provisioning or resource allocation and other is task scheduling in distributed environment. Efficient provisioning of resources and scheduling of resources as well as tasks will ensure [9]:

a. Resources are easily available on demand.

b. Resources are efficiently utilized under condition of high/low load.

c. Energy is saved in case of low load (i.e., when usage of cloud resources is below certain threshold).

d. Cost of using resources is reduced.

For measuring the efficiency and effectiveness of Load Balancing algorithms simulation environment are required [6].

\subsection{Resource Allocation}

Resource provisioning is the job of mapping of the resources to different entities of cloud on demand basis. Resources must be allocated in such a manner that no node in the cloud is overloaded and all of the available resources in the cloud do not undergo any type of wastage (wastage of bandwidth or processing core or memory etc.). 


\subsection{Task Scheduling}

Task scheduling is done after the resources are allocated to all cloud entities. Scheduling defines the manner in which different entities are provisioned [10]. Resource provisioning defines which resource is going to be available to meet user requirements whereas task scheduling defines the manner in which the allocated resource is available to the end-user (i.e., if the resource is fully available until task completion or is available on sharing basis). Task scheduling provides "Multiprogramming Capabilities" in cloud computing environment. Task scheduling can be achieved in two modes: a. Space shared $b$. Time shared

Both hosts and VM can be provisioned to users either in space shared mode or time shared mode. In space sharing mode resources are allocated until task does not undergo complete execution (i.e., resources are not pre-empted); whereas in time sharing mode resources are continuously pre-empted till task undergoes completion.

\subsection{Load Balancing on the Cornerstone of Cloud Environment}

Cloud computing might have either static or dynamic environment in relation to how developer configures the cloud demanded by the cloud provider.

\subsubsection{Static Environment}

In static environment the cloud provider installs homogeneous resources. Also the resources in the cloud are not flexible when environment is made static. In this scenario, the cloud requires prior familiarity with nodes capacity, processing power, memory, performance and statistics of user requirements. These user requirements are not subjected to any change at run-time.

\subsubsection{Dynamic Environment}

In dynamic environment the cloud provider installs heterogeneous resources. The resources are flexible in dynamic environment. In this scenario cloud cannot rely on the last knowledge whereas it will take into account run-time statistics. The requirements of the users are granted flexibility (i.e., they may change at run-time). Algorithm proposed to achieve load balancing in dynamic environment can quickly adapt to run time changes in load. Dynamic environment is difficult to be simulated but is highly adaptable with cloud computing environment [9].

\section{Literature Survey}

Juan, Chen, et al., [1] presented spectral properties of some different realworld networks such as for example as example regular networks, random networks, small-world networks, scale-free networks, and so on. They unearthed that in random networks, the littlest nonzero eigen value grows approximately linearly with respect to the probability $\mathrm{p}$. Consequently with this specific, some estimates for the littlest nonzero eigen values of random networks could possibly be obtained. More interestingly, it absolutely was shown a great correlation relating to the Eigen value spectrum and degree sequence in the networks, especially in scale-free networks. Using this correlation, they developed a nearby algorithm to find out the Eigen value $\lambda_{\mathrm{i}+1}$ from $\lambda_{\mathrm{i}}$.

Guo-hong, et al., [2] indicated that there surely is an thorough relation relating to the evolution mechanism of cluster innovation networks and the risk-resistant capability of the networks, and the process of evolving on the foundation of the principle of "preferential attachment" brings in relation to the scale-free character of the cluster innovation networks; the uniform distribution of enterprises strength contributed to enhance the invulnerability of the innovation networks, there is a threshold of inter-firm 
connection density that intensive inter-firm relationship plays a role in the risk-resistant ability of innovation networks up to and including threshold and beyond which more intensive inter-firm relationship can result in a decline in risk-resistant ability of innovation networks. Finally, it absolutely was unearthed that the key element factors inducing the cascading breakdown of cluster innovation networks could possibly be summarized into three areas: the distribution of inter-firm connections, the distribution of firms 'risk-resistant capabilities, and the innovation capabilities of the enterprises within the cluster. Some recommendations regarding to improving the robustness of cluster innovation networks were proposed to be able to avoid the occurrence of cascading breakdown in industrial cluster.

Chen, Pin-Yu, et al., [3] investigated the IDD in well-known complex networks by modified Susceptible-Infected (SI) model, that's been surprisingly suitable for above multiple scenarios. Systematically categorizing such networks and examining conditions to adopt SI model for IDD in complex networks, the fundamental properties including mixing type, vertex connectivity and giant component size provide valid insights for quantitative analysis. In addition they investigated the IDD in networks with time-varying topology and reveal that individuals receive the info in dynamic sense, even if the giant component size wasn't appropriate for just how many individuals in static sense. Consequently, they successfully established such analytical model for characterizing the IDD in complex networks consisting of opportunistic links and time-varying topology, feasible for various random wireless networks and social networks.

Li, Gang, et al., [4] introduced a story topological structure evolving model of complex supply networks from the perspective of Complex Networks. After investigating supply networks typical structural characteristics and formation mechanisms, Complex Supply Networks Evolving Model (CSNEM) are proposed to mimic these structural characteristics. Then, three statistical properties were investigated. To validate CSNEM, three real supply chain networks are introduced. The end result indicated that CSNEM was in line with the real-world supply networks. Further the relations between these properties and supply networks macro behaviours i.e., rapid response capability and robustness are discussed. This work would provide managers and researches a beneficial insight on macroscopic structures characteristics plus a helpful understanding on the relations between macroscopic structures and global behaviours in supply networks.

Hu, Ziping, et al., [5] presented some of the characteristics of complex networks that affect their performance. Complex networks in this paper were typified as random networks, small-world networks, and scale-free networks. In addition to discussing some of the generic properties of the networks, this paper dedicated to a comparison on the set of networks because of their resilience against failure and attack. The small-world (WS) network was found to genuinely have the most effective resilience in comparison to others due to the highly clustered feature. The outcomes help one in gaining a greater comprehension of the field.

Yijun, Zhang, et al., [6] discussed the design problem of global asymptotical synchronization through adaptive control for a type of complex networks with timevarying delayed couplings. In the complex networks under consideration, the dynamic of every node was expressed with a neutral-type system with time-varying delays. Using the LaSalle invariance principle and some properties of Kronecker product, a sufficient condition that globally asymptotically stabilizes the error system through adaptive control is derived, which ensures the dynamics of whole networks synchronize with the desirable synchronization manifold. In the proposed criterion, the matrices denoting the topological structure of the complex networks are not must be symmetric. And the adaptive feedback strength was updated predicated on both todays and the delayed state errors of the networks. 
A numerical example was presented to exhibit the effectiveness and applicability of the proposed approach.

Li, Xiang, et al., [7] studied the pioneering studies of small-world and scalefree networks in the late 1990 they've witnessed fruitful advances in comprehension of the complex connection properties and characteristics of numerous diverse large-scale distributed interconnected systems, both natural and man-made, with examples which range from the Internet, the World Wide Web, biological neural networks, protein-toprotein interaction networks, power-grids, wireless communication networks to social, economic and financial networks in human society. Extensive efforts have previously been focused on characterizing the rich connectivity patterns on the list of nodes (components) of such complex networks (systems), and in the span of development of research of this type, people have previously been prompted to deal with on a fundamental question: Just how can the fascinating yet complex topological features of a network affect or determine the collective behavior and performance of the networked system.

Chira, Camelia, et al., [8] proposed a fresh fitness function for the assessment of community structures quality which may be on the foundation of the amount of nodes and their links in the residential district versus the city size further reported to how big the network is. A story area of the proposed fitness function refers to thinking about the manner in which nodes hook up to other nodes inside the exact same community causing the 2 nd amount of links donate to the potency of the community. The introduced fitness function is tested in a very collaborative evolutionary algorithm created designed for the problem of community detection in complex networks. Computational experiments were performed for numerous real-world complex networks which have a known real community structure. This allowed the direct verification of the grade of evolved communities via the proposed fitness function emphasizing extremely promising numerical results.

Jin, et al., [9] discussed development of economy and information technology has increased the scale of aviation networks and enhanced the complex interaction in the network. Therefore the analysis of aviation networks is among probably the most hot research issue in the international research academy. The complex networks theory that will be highly abstracted from complex system has been researched thoroughly. Thus, it absolutely was used here to analyze the structure of aviation networks. To start with, the topological options that come with complex aviation networks were analyzed. Then a spatial distribution of Chinese aviation network is researched consistent with the indices of degree, clustering coefficient, accessibility, and so on. At last, the analysis revealed that Chinese aviation network is a "Small World" networks whose center may be the airports with large degree. The network analysis model proposed here directed the improvement of Chinese aviation network development.

Liu, Yong-Chun, et al., [10] studied adaptive synchronization in regularly coupled complex dynamical networks. An adaptive coupling strength algorithm was proposed to effectively stabilize and synchronize complex networks. The strictness proof of this algorithm from the viewpoint of mathematics was given. Especially, they revealed that synchronization of such complex dynamical networks is wholly determined by the coupling strength. An example was simulated, using the chaotic Lorenz system, since the nodes of the complex dynamical networks, which demonstrate the potency of the proposed adaptive algorithm.

Zhou, Yuehua, et al., [11] showed a book framework of the fault and intrusion tolerance of complex networks in a controllability view, which took the number of driver nodes as standard. The result, the SF networks had a better fault tolerance compared to ER networks, is consistent with the result considered with available researches. However the SF networks and ER networks have poor intrusion tolerance. The key reason why was so it was more challenging to totally control the real SF networks than to totally control the 
real ER networks, so it absolutely was easier to thoroughly destroy the controllability of the real SF networks compared to real ER networks.

Wang, Yujie, et al., [12] modeled and evaluate the reliability of scale-free complex networks utilizing a binary decision diagram (BDD)-based method. Two-terminal reliability is called, which will be defined since the probability a specified couple of nodes can communication through at the least one fault-tree path in the network. This reliability metric can measure, as an example, how reliable the data may be shared between two parties or most of the time how reliable the data may be disseminated within a social network.. The Barabási-Albert model was useful for generating the sample scalefree networks studied in this paper. The reliability performance of scale-free networks is in comparison to that particular of random networks beneath the Erdos-Renyi model. Degree distributions and clustering coefficients (average fraction of pairs of neighbors of a node which may also be neighbors of every other) of sample scale-free and random networks are illustrated and compared. Their effects on the performance of the BDD-based method for the reliability analysis of both scale-free and random networks are likewise discussed.

M. Katyal, A. Mishra [13] analyzed that Cloud Computing is often a new trend emerging in IT environment with huge requirements of infrastructure and resources. Load Balancing is a significant aspect of cloud computing environment. Efficient load balancing scheme ensures efficient resource utilization by provisioning of resources to cloud user's on-demand basis in pay-as-you-say-manner. Load Balancing will even support prioritizing users by using appropriate scheduling criteria.

Li, Yun, et al., [14] proposed an algorithm for overlapping detection in complex networks based on the boundary information of disjoint community. For a boundary node, it connects several disjoint communities. The algorithm computed the sharpness variations of the boundaries attributable to the boundary node. It judges perhaps the boundary node belongs to multi-communities synchronously. A overlapping nodes on the list of disjoint communities can be found. Moreover, the overlapping ratio of complex networks can be controlled by parameter of the algorithm. This is helpful to reveal the overlapping communities of complex networks hierarchically with a extent. In experimental tests utilizing a synthetic random network and three real networks, the algorithm was proved to be simple and feasible, and it could detect overlapping communities in complex networks flexibly and effectively.

Youssef, Bassante, et al., [15] proposed a generation model for heterogeneous complex networks. They introduced novel model "settling node adaptive model" SNAM. SNAM reflects the heterogeneous nature of nodes connection-standard requirements. Such novel nodes connection standard criterion wasn't an integral part of any previous network generation models. SNAM was successful in preserving the ability law degree distribution, the small world phenomenon and the high clustering coefficient of complex networks.

Ze links, Ivan, et al., [16] demonstrated that dynamics of evolutionary algorithms that were centered on Darwin theory of evolution and Mendel theory of genetic heritage can be also visualized as complex networks. Such network might be then analyzed through classical tools of complex networks science. Results presented were currently numerical demonstration rather than theoretical mathematical proofs. They open question whether evolutionary algorithms really create complex network structures and whether this knowledge might be successfully used like feedback for control of evolutionary dynamics and its improvement in order to raise the performance of evolutionary algorithms.

Curia, Vincenzo, et al., [17] proposed a new approach for modeling Wireless Sensor Networks (WSNs with the target of ensuring a marked improvement in regards to clustering coefficient and average network diameter. With the proposed hybrid idea, the major section of nodes wouldn't be necessarily adjacent but, for every pair of the, there 
would exist a comparatively short path for a stable connection. The potency of the proposed idea had been validated by way of a heavy campaign of simulations.

\section{Gaps in Literature}

1. The all-around nodes may not gain highest score in a few heuristics for cloud computing.

2. The heuristic approaches for cloud computing are very time consuming and not scalable in nature.

3. The usage of complex networks has been neglected for cloud computing by most researchers.

So using the complex networks in cloud computing to optimize cloud services is the key motivation of this research work.

\section{Conclusion and Future Scope}

Complex networks have become extremely popular way to resolve many networking related problems within a proficient manner. In this paper, from the review, it's been found that the all-around nodes may not gain highest score in a few heuristics for cloud computing. Moreover the heuristic approaches for cloud computing are very time consuming and not scalable in nature. Other issue is that the utilization of complex networks has been neglected for cloud computing by most researchers. So in not too distant future, the complex networks will undoubtedly be used for cloud computing environment to optimize the cloud web services further.

\section{References}

[1] C. Juan and L. Jun-An, "Laplacian spectral properties of complex networks. In Control Conference (CCC)", 2010 29th Chinese, IEEE, (2010), pp. 4684-4689. IEEE.

[2] W. Guo-Hong, X. Rui, T. Li-Yan and S. Kittisarawanno, "The risk of cascading breakdown in industrial cluster innovation networks: a complex networks perspective", In Management Science and Engineering (ICMSE), 2010 International Conference on IEEE, (2010), pp. 1445-1455.

[3] P.-Y. Chen and K.-C. Chen, "Information epidemics in complex networks with opportunistic links and dynamic topology", In Global Telecommunications Conference (GLOBECOM 2010) IEEE, (2010), pp. $1-6$.

[4] G. Li, Q. Xuan, Z. Song and X. Jin, "Complex supply networks evolving model: Complex networks perspective", In Intelligent Systems and Knowledge Engineering (ISKE), 2010 International Conference on IEEE, (2010), pp. 511-516.

[5] Z. Hu and P. K. Verma, "Topological resilience of complex networks against failure and attack", In Advanced Networks and Telecommunication Systems (ANTS), 2011 IEEE 5th International Conference on IEEE, (2011), pp. 1-6.

[6] Z. Yijun and Q. Yi, "Adaptive synchronization of complex networks with neutral-type nodes", In Control Conference (CCC), 31st Chinese, IEEE, (2012) pp. 1195-1200.

[7] X. Li, D. Yang, X. Liu and X-M. Wu, "Bridging Time Series Dynamics and Complex Network Theory with Application to Electrocardiogram Analysis", Circuits and Systems Magazine, IEEE 12, no. 4, (2012), pp. 33-46.

[8] C. Chira, A. Gog and D. Iclanzan, "Evolutionary detection of community structures in complex networks: A new fitness function", In Evolutionary Computation (CEC), Congress on IEEE, (2012), pp. $1-8$.

[9] J. He, Z. Liu, Y. Wu and S. Li, "Analysis of Aviation Networks Structure Based on Complex Networks Theory", In Proceedings of the 2012 Second International Conference on Instrumentation, Measurement, Computer, Communication and Control, IEEE Computer Society, (2012), pp. 179-182.

[10] Y.-C. Liu and D. Lin, "Adaptive synchronization on complex dynamical networks", In Biomedical Engineering and Informatics (BMEI), 2012 5th International Conference on IEEE, (2012), pp. 12321235.

[11] Y. Zhou, Y. Zeng, Z. Liu, N. Li, J. Ma and L. Dong, "Fault and Intrusion Tolerance of Complex Networks: A Controllability View", In Intelligent Networking and Collaborative Systems (INCoS), 2013 5th International Conference on IEEE, (2013), pp. 516-520.

[12] Y. Wang, L. Xing and H. Wang, "Reliability of scale-free complex networks. In Reliability and Maintainability Symposium (RAMS)”, 2013 Proceedings-Annual, IEEE, (2013), pp. 1-6. 
[13] M. Katyal and A. Mishra, "A Comparative Study of Load Balancing Algorithms in Cloud Computing Environment", International Journal of Distributed and Cloud Computing, vol. 1, Issue 2, (2013) December.

[14] Y. Li, G. Liu and S.-Y. Lao, "Overlapping community detection in complex networks based on the boundary information of disjoint community", In Control and Decision Conference (CCDC), 2013 25th Chinese,IEEE, (2013), pp. 125-130.

[15] B. E. Youssef and M. R. M Rizk, "SNAM: A heterogeneous complex networks generation model", In Heterogeneous Networking for Quality, Reliability, Security and Robustness (QShine), 2014 10th International Conference on IEEE, (2014), pp. 44-50.

[16] I. Zelinka, D. Davendra, J. Lampinen, R. Senkerik and M. Pluhacek, "Evolutionary algorithms dynamics and its hidden complex network structures", In Evolutionary Computation (CEC), Congress on IEEE, (2014), pp. 3246-3251.

[17] V. Curia, M. Tropea, P. Fazio and S. Marano, "Complex networks: Study and performance evaluation with hybrid model for Wireless Sensor Networks", In Electrical and Computer Engineering (CCECE), 2014 IEEE 27th Canadian Conference on, (2014), pp. 1-5. 
International Journal of Hybrid Information Technology

Vol.8, No.3 (2015) 